\title{
Avaliação de métodos de amostragem de "Cascudinhos" Alphitobius diaperinus (Panzer) (Coleoptera: Tenebrionidae) em cama de frangos de corte
}

\section{Evaluations of sampling methods for "Darkling beetles" (Alphitobius diaperinus Panzer) (Coleoptera: Tenebrionidae) in the litter of broiler houses}

\author{
Giane Serafim da Silva1,2; Viviane Aparecida Veronez'; Gilson Pereira de Oliveira'; \\ Fernando de Almeida Borges ${ }^{1}$; Heloísa Cristina da Silva ${ }^{1}$; Marcelo Vasconcelos Meireles ${ }^{3}$
}

\begin{abstract}
Resumo: O Alphitobius diaperinus tem se apresentado mundialmente como uma das principais pragas da avicultura moderna. Com a expansão avícola industrial, este coleóptero conhecido como cascudinho encontrou junto às instalações avícolas habitat ideal para seu desenvolvimento. Adultos, ovos, larvas e pupas são encontrados sob a superfície da cama, em equipamentos e em frestas dos pisos e paredes, podendo causar inúmeros prejuízos. O monitoramento da população de $A$. diaperinus, utilizando métodos quantitativos de amostragem, é de fundamental importância para o estabelecimento de medidas de controle e para avaliação da eficácia de medicamentos. O presente estudo foi conduzido no aviário experimental do "Centro de Pesquisas em Sanidade Animal" - CPPAR - FCAVJ/UNESP, onde foram avaliados dois métodos de quantificação de A. diaperinus: armadilhas modificadas de Arends e Tijolos, para contagem de cascudinhos presentes em cama de frango. Pelos resultados obtidos, em 192 contagens para cada método empregado, pode-se inferir que os dois métodos de amostragem não se diferenciaram $(P>0,05)$ no que se refere à avaliação de infestação por adultos e larvas de $A$. diaperinus.
\end{abstract}

Palavras-chave: cascudinho, Alphitobius diaperinus, cama de frango, armadilhas.

Abstract: The Alphitobius diaperinus has been globally one of the major ectoparasites of the modern poultry production. With the industrial poultry expansion, this coleopteran has found in poultry facilities an excellent habitat for its development. Adults, eggs, larvae and pupae are found under the litter surface, in equipment and in cracks of the floors and walls, where they can cause remarkable damages. The counting of the $A$. diaperinus population using quantitative methods of sampling is of fundamental importance for the establishment of appropriate control measures and for evaluation of the effectiveness of insecticides. The present study was carried out in the experimental aviary of the "Center of Researches in Animal health" - CPPAR - FCAVJ/UNESP, where two methods of quantification of A. diaperinus were evaluated: the method of modified traps of Arends and the method of bricks, for counting of $A$. diaperinus in the chicken little. It was concluded that the two sampling methods employed did not differ $(P>0,05)$ in the evaluation of infestation for adults and larvae of $A$. diaperinus.

Key words: darkling beetles, Alphitobius diaperinus, litter, broiler, traps.

\section{Introdução}

O Alphitobius diaperinus, considerado inicialmente uma praga secundária de grãos armazenados (LEGNER e OLTON, 1970), tem se apresentado mundialmente como uma das principais pragas da avicultura moderna (ARENDS, 1987; STEELMAN, 1996; PAIVA, 2000). Com a expansão avícola industrial, este coleóptero pertencente à família Tenebrionidae e popularmente conhecido como "cascudinho", encontrou, junto às instalações avícolas, habitat ideal para seu desenvolvimento.

Uma vez estabelecidos, multiplicam-se rapidamente (KHAN et al., 1998), sendo encontradas elevadas populações em cama de frangos de corte, de matrizes, e mesmo em fezes de poedeiras de ovos comerciais confinadas em gaiolas (MATIAS, 1995). Adultos, ovos, larvas e pupas vivem sob a superfície da cama, em equipamentos e em frestas dos pisos e paredes (PAIVA, 2000); alimentam- se de ração, aves mortas, aves debilitadas, esterco e outros materiais orgânicos em decomposição encontrados no galpão.

Além desses aspectos, considerados injuriáveis, seus prejuízos estão relacionados à capacidade de atuarem como reservatório de patógenos, e exemplo de vírus, bactérias, fungos e protozoários (HOFSTAD et al., 1972; SAFRIT e AXTELL, 1984; CRAWFORD et al., 1998); de serem classificados como hospedeiros intermediários de cestódeos parasitos de aves (ARENDS, 1987); de interferirem no desenvolvimento das aves causando desuniformidade do lote (MATIAS, 2000) e por danificarem os equipamentos, determinando sérios prejuízos nas instalações avícolas (TURNER, 1986).

O monitoramento da população de $A$. diaperinus, utilizando-se métodos quantitativos de amostragem, é de fundamental importância para o estabelecimento de

${ }^{1}$ CPPAR-Centro de Pesquisas em Sanidade Animal, FCAVJ/UNESP, Campus de Jaboticabal

2 UNICASTELO - Universidade Camilo Castelo Branco - Campus VIII, Descalvado

${ }^{3}$ FOA/UNESP - Curso de Medicina Veterinária, Campus de Araçatuba 
adequadas medidas de controle do $A$. diaperinus e para avaliação da eficácia de medicamentos adotados no seu tratamento. A literatura internacional tem mostrado o uso de armadilhas para captura de formas adultas e imaturas do cascudinho (SAFRIT e AXTELL, 1984), sendo o modelo de Arends e o modelo modificado de Arends os mais empregados (STRINGHAM e ARENDS, 2000; STRINGHAM, 2000).

Dois métodos de quantificação de $A$. diaperinus foram avaliados neste trabalho: $O$ uso de armadilhas modificadas de Arends (com abertura de apenas uma das extremidades) e a disposição de Tijolos em vários locais da cama de frango da instalação, das quais foram recolhidos cascudinhos.

\section{Material e Métodos}

O estudo foi conduzido no aviário experimental do "Centro de Pesquisas em Sanidade Animal" (CPPAR FCAVJ/UNESP), que é provido de 20 boxes de $3,6 \mathrm{~m}^{2}$ cada, separados por muretas de alvenaria e tela. Cada boxe possui, individualmente, bebedouros, comedouros e sistemas de aquecimento para aves.

Após um ciclo de produção, cama de frango (casca de arroz) infestada por $A$. diaperinus foi retirada de galpões comerciais de frangos de corte e transportada para o aviário experimental do CPPAR, sendo homogeneamente distribuída em 04 boxes. Decorrido o período de adaptação, ou seja, três dias da distribuição da cama infestada, a presença de cascudinho foi confirmada por meio de amostragens realizadas em vários locais dentro de cada boxe (D0). Uma vez confirmada a infestação, os boxes foram sorteados para a constituição das parcelas experimentais.

Pintos de corte da linhagem COBB, com um dia de idade, foram alojados em cada boxe, observando a mesma densidade. As aves receberam água à vontade e ração formulada de acordo com as exigências nutricionais, adotando-se manejo usual em avicultura.

\subsection{Modelo modificado de Arends}

Para o primeiro método avaliacional, foram preparadas armadilhas seguindo o modelo modificado de Arends (STRINGHAN e ARENDS, 2000). Utilizou-se tubos de PVC (polivinilcloridro) de $3,8 \mathrm{~cm}$ de diâmetro por $23 \mathrm{~cm}$ de comprimento, com uma abertura longitudinal de $0,65 \mathrm{~cm}$ e contendo papel microondulado $(20 \mathrm{~cm} \times 30 \mathrm{~cm})$ enrolado em seu interior; apenas uma das extremidades foi fechada.

Seis armadilhas foram colocadas sob a cama de frango, em diferentes locais dentro de cada boxe (seis repetições/parcela), totalizando 24 armadilhas. A abertura longitudinal ficava voltada para o lado inferior, em direção ao piso, sendo totalmente coberta pela cama de frango. Em intervalos semanais o conteúdo de cada armadilha era recolhido e o número de adultos e de larvas de $A$. diaperinus contado. Os coleópteros pertencentes a cada parcela, após contabilização, eram devol- vidos em cada boxe para que não houvesse interferência na população de $A$. diaperinus. As avaliações foram realizadas em intervalos semanais até o 49 dia experimental $(0,7,14,21,28,35,42,49 \mathrm{DE})$, data em que se encerrou o experimento.

\subsection{Tijolos sobrepostos à cama de frango}

O segundo método de avaliação foi a distribuição de tijolos, em diferentes locais, sobre a cama de frango. Semanalmente eram colhidos conteúdos cama, contendo larvas e adultos de cascudinhos, que se encontravam alojados sob os tijolos. Cada amostra era acondicionada em recipiente plástico com tampa perfurada e transportada até o laboratório para a realização das contagens. Após homogeneização, eram pesados 100 gramas do conteúdo recolhido, padronizando-se as amostras. As datas e período de avaliação assim como os procedimentos após contagem foram os mesmos adotados para armadilhas modificadas de Arends $(0,7,14,21$, 28, 35, 42, 49 DE) .

\subsection{Necropsia parasitológica}

Neste trabalho procurou-se, ainda, confirmar a ingestão de $A$. diaperinus pelos pintinhos jovens. Neste sentido, após um, três e cinco dias do alojamento das aves nos boxes, três aves de cada parcela foram sacrificadas e submetidas à necropsia parasitológica. Foi pesquisada, sob estereomicroscopia, a presença de adultos e de larvas no conteúdo do trato digestivo das aves (papo, proventrículo e moela).

\subsection{Delineamento experimental}

O delineamento foi em parcela subdividida no tempo ("Split Plot in Time"), sendo considerados, como tratamentos principais, os métodos de avaliação e como secundário as datas de observações (BANZATTO e KRONKA, 1989). As análises dos dados foram realizadas aplicando-se o teste $\mathrm{F}$ e as médias (dados transformados em log $(x+1))$ comparadas pelo teste de Tukey (SAS INSTITUTE, 1989).

\section{Resultados e Discussão}

Os métodos de amostragem empregados, armadiIhas modificadas de Arends e Tijolos, não se diferenciaram, estatisticamente $(P>0,05)$, durante todo o ciclo de produção das aves (1-49 dias) (Tabelas 1 e 2), no que se refere à avaliação de infestações por adultos e larvas de A. diaperinus. Apenas no dia zero, para confirmação da infestação, momento em que as aves ainda não tinham sido alojadas, o método de Tijolos apresentou maior número de cascudinhos colhidos, sejam adultos ou larvas $(P<0,05)$. A conveniência da utilização de armadilhas para captura de cascudinhos, seguindo os modelos de Arends e/ou modelo modifica-do de Arends tem sido comprovada pela literatura (SAFRIT e AXTELL, 1984; STRINGHAM e ARENDS, 2000; STRINGHAM, 2000). A utilização do modelo Tijolos na amostragem dos coleópteros não foi ainda relatada na literatura e, 
embora mais simples, mostrou-se igualmente eficaz na avaliação da população de A. diaperinus. Por outro lado, tal método revelou-se mais trabalhoso, pois o volume a ser inspecionado é bem maior do que o colhido pelo modelo modificado de Arends.

Adicionalmente, verificou-se neste estudo que o número de larvas e adultos amostrados no $49^{\circ}$ dia foi superior ao avaliado no dia zero, mostrando que houve proliferação dos coleópteros no ambiente; a relação adultos/larvas foi maior no dia zero em relação aos dados do 49 dia, caracterizando reprodutividade.

O conhecimento de particularidades biológicas deste inseto é de suma importância no estabelecimento de programas de controle de $A$. diaperinus. Insere-se neste aspecto a escolha do fármaco, pois ficou demonstrado que o número de larvas aumenta consideravelmente em relação ao número de adultos e, dentre os medicamen- tos, existem aqueles com maior atuação do princípio ativo em larvas ou em adultos.

Os achados de necropsia, ou seja, adultos e larvas de $A$. diaperinus presentes no papo, proventrículo e moela das aves, nas idades estudadas, mostraram não ter havido ingestão significativa de cascudinhos nos primeiros dias de criação (Tabela 3). Estudos detalhados, especialmente delineados para tal comprovação, devem ser executados, uma vez que a literatura cita interferência da infestação e ingestão de cascudinhos no desempenho das aves.

\section{Agradecimentos}

Os autores agradecem ao Dr. Mike Stringham, Universidade do Estado da Carolina do Norte, pela gentileza e disponibilização de informações.

Tabela 1 - Comparação múltipla do número médio * (dados transformados em $(\log x+1)$ ) de adultos de Alphitobius diaperinus, avaliados pelos modelos "Tijolos" e "Armadilhas modificadas de Arends". CPPAR. Jaboticabal, SP, Brasil.

\begin{tabular}{|c|c|c|c|c|c|c|c|c|}
\hline \multirow{2}{*}{ Grupos } & \multicolumn{8}{|c|}{ N Médio de Adultos de A. diaperinus / Dias de Avaliação } \\
\hline & 0 & 7 & 14 & 21 & 28 & 35 & 42 & 49 \\
\hline Tijolos & $1,5782 \mathrm{a}$ & $0,6349 \mathrm{a}$ & $1,2902 \mathrm{a}$ & $1,0294 \mathrm{a}$ & $0,9823 \mathrm{a}$ & $0,9604 \mathrm{a}$ & $1,2637 \mathrm{a}$ & $1,6570 \mathrm{a}$ \\
\hline Armadilhas & $0,1266 \mathrm{~b}$ & $0,2448 a$ & $0,7997 \mathrm{a}$ & $0,8821 \mathrm{a}$ & $0,7583 a$ & $0,8411 a$ & $1,3583 \mathrm{a}$ & $1,3578 \mathrm{a}$ \\
\hline
\end{tabular}

***Médias seguidas pela mesma letra, na coluna, não diferem entre si pelo Teste de Tukey $(P>0,05)$

Tabela 2 - Comparação múltipla do número médio * (dados transformados em $(\log \mathrm{x}+1)$ ) de larvas de Alphitobius diaperinus, avaliados pelos modelos "Tijolos" e "Armadilhas modificadas de Arends". CPPAR. Jaboticabal, SP, Brasil.

\begin{tabular}{|c|c|c|c|c|c|c|c|c|}
\hline \multirow{2}{*}{ Grupos } & \multicolumn{8}{|c|}{ № Médio de Larvas de A. diaperinus / Dias de Avaliação } \\
\hline & 0 & 7 & 14 & 21 & 28 & 35 & 42 & 49 \\
\hline Tijolos & $1,7144 \mathrm{a}$ & $1,1634 \mathrm{a}$ & 1,9431 a & $2,6600 \mathrm{a}$ & $2,5065 \mathrm{a}$ & 2,4057 a & 2,3429 a & $2,4664 \mathrm{a}$ \\
\hline Armadilhas & $0,3178 \mathrm{~b}$ & $0,7248 \mathrm{a}$ & $1,6920 \mathrm{a}$ & $2,7270 \mathrm{a}$ & $2,6563 a$ & $2,5297 \mathrm{a}$ & $2,4793 \mathrm{a}$ & $2,3486 \mathrm{a}$ \\
\hline
\end{tabular}

\footnotetext{
***Médias seguidas pela mesma letra, na coluna, não diferem entre si pelo Teste de Tukey $(P>0,05)$
} 
Tabela 3 - Adultos e larvas de Alphitobius diaperinus, encontrados em aves necropsiadas nos dias 1, 3 e 5 pós alojamento. CPPAR, Jaboticabal, SP, Brasil.

\begin{tabular}{ccccccc}
\hline \multirow{2}{*}{ Repetição } & \multicolumn{6}{c}{$\mathbf{N}^{\circ}$ de A. diaperinus / Datas das necropsias* } \\
\cline { 2 - 7 } & $\mathbf{1}$ & $\mathbf{3}$ & $\mathbf{5}$ & $\mathbf{1}$ & $\mathbf{3}$ & $\mathbf{5}$ \\
\cline { 2 - 7 } & 1 & 0 & 0 & 0 & 0 & 0 \\
$\mathbf{1}$ & 0 & 1 & 0 & 0 & 0 & 0 \\
$\mathbf{3}$ & 0 & 0 & 0 & 0 & 0 & 0 \\
$\mathbf{4}$ & 0 & 0 & 0 & 0 & 0 & 1 \\
\hline
\end{tabular}

*: Dias pós alojamento das aves

\section{Referências Bibliográficas}

ARENDS, J.J. Control, management of the litter beetle. Poultry Digest., Mount Morris, v.44, p.172-176, 1987.

BANZATTO, D. A; KRONKA, S. N. Experimentação Agrícola. Jaboticabal: FUNEP, 1989.

CRAWFORD, P.J.;BROOKS, W.M.; ARENDS, J.J. Efficacy of field-isolated strains of Beauveria bassiana (Moniliales: Moniliaceae) as microbial control agents of the lesser mealworm (Coleoptera:Tenebrionidae). Journal of Economic Entomology., College Park, v. 91, n.6, p1295-1301, 1998.

HOFSTAD, M.S.; CALNECK, B.W.: HELMBOLT, C.F.; REID, W.M.; YODER, H.W.J. (Eds.) Diseases of poultry. Ames: lowa State University Press, 1972.

KHAN, B.A.; DAY, P.A.; GOONEWARDENE, L.A.; ZUIDHOF, M.J.; HAWKINS, G. Efficacy of tetrachlorvinphos insecticide dust against darklig beetles in commercial broiler chicken barns. Canadian Journal of Animal Science., Champaign, v.78, n.4, p.723-725, 1998.

LEGNER, E.F.; OLTON, G.S. Worldwide survey and comparison of adult predatorad scavenger insect populations associated with domestic animal manure where livestock is artificially congregated. Hilgardia, Berkeley, n. 40, p.225256, 1970.

MATIAS, R. S. Cascudinho. In: CONFERÊNCIA APINCO DE CIÊNCIA E TECNOLOGIA AVÍCOLAS, 1995, Curitiba. Anais... Curitiba: FACTA, 1995.

MATIAS, R. S. O Controle do cascudinho: novas perspectivas. In: SIMPÓSIO BRASIL SUL DE AVICULTURA, 1, 2000, Chapecó. Anais... Chapecó: [s.n.], 2000. p.169-71.

PAIVA, D.P. Cascudinhos: biologia. SIMPÓSIO BRASIL SUL DE AVICULTURA, 1, 2000, Chapecó. Anais... Chapecó: [s.n.], 2000. p. 135-139,

SAFRIT, R.D.; AXTELL, R.C. Evaluations of sampling methods for darkling bettles (Alphitobius diaperinus) in the little of turkey and broiler houses. Poultry Sci., Champaign, v.63, p.2368-2375, 1984.

SAS INSTITUTE. User's guide: statistics. Cary, 1989.

STEELMAN, D. Darkling beetless are costly pests. Poultry Digest, Mount Morris, v.55, n.10, p.22-23, 1996.
STRINGHAM, S. M. Comparison of standard and modified tube traps for sampling darkling beetles, Alphitobius diaperinus (Panzer), in broiler houses. [mensagem pessoal]. Mensagem recebida por <serafim@fcav.unesp.br> em 08 dez. 2000. (Not for publication)

STRINGHAM, M.; ARENDS J.J. Evaliation of a modified Arends tube trap for sampling the lesser mealworm, Alphitobius diaperinus (Panzer), in turkey brooder houses. [mensagem pessoal]. Mensagem recebida por <serafim@ fcav.unesp.br> em 08 dez. 2000. (Not for publication)

TURNER JR, E.C. Structural ad litter pests. Poultry Science, Champaign, v.65, p.644-648, 1986. 